UMN-TH-1829/99

TPI-MINN-99/54

OUTP-99-62P

hep-ph/9912266

December 1999

\title{
Single-Brane Cosmological Solutions with a Stable Compact Extra Dimension
}

\author{
Panagiota Kanti ${ }^{1}$, Ian I. Kogan ${ }^{2}$, Keith A. Olive ${ }^{1}$ and Maxim Pospelov ${ }^{1}$ \\ ${ }^{1}$ Theoretical Physics Institute, School of Physics and Astronomy, \\ University of Minnesota, Minneapolis, MN 55455, USA \\ ${ }^{2}$ Theoretical Physics, Department of Physics, Oxford University \\ 1 Keble Road, Oxford, OX1 3NP, UK
}

\begin{abstract}
We consider 5-dimensional cosmological solutions of a single brane. The correct cosmology on the brane, i.e., governed by the standard 4-dimensional Friedmann equation, and stable compactification of the extra dimension is guaranteed by the existence of a nonvanishing $\hat{T}_{5}^{5}$ which is proportional to the 4 -dimensional trace of the energy-momentum tensor. We show that this component of the energy-momentum tensor arises from the backreaction of the dilaton coupling to the brane. The same positive features are exhibited in solutions found in the presence of non-vanishing cosmological constants both on the brane $\left(\Lambda_{b r}\right)$ and in the bulk $\left(\Lambda_{B}\right)$. Moreover, the restoration of the Friedmann equation, with the correct sign, takes place for both signs of $\Lambda_{B}$ so long as the sign of $\Lambda_{b r}$ is opposite $\Lambda_{B}$ in order to cancel the energy densities of the two cosmological constants. We further extend our single-brane thin-wall solution to allow a brane with finite thickness.
\end{abstract}




\section{Introduction}

It goes without saying that there has been an exceptional amount of interest in theories with extra dimensions, and in particular the resulting cosmologies that arise in those theories. In the past, in theories with extra dimensions, one often simply assumed a small compact extra dimension, of order the Planck size. In this case, it is usually sufficient to average over the extra dimension(s) and once fixed, cosmology on the large 4D space-time is conventional, other than the appearance of additional massive degrees of freedom. In $10 \mathrm{D}$ string theory, while the structure of the compact $6 \mathrm{D}$ space has a profound effect on the resulting interactions of matter in the effective low-energy theory, cosmology is only affected by terms suppressed by inverse powers of the Planck scale. Below the Planck scale, these are generally negligible. When string theory is promoted to M-theory, the size of the extra 11th dimension, is generally assumed to be somewhat larger than $M_{P}^{-1}$, and thus allows for a separation of the string (unification) scale and the Planck scale [1].

By allowing the extra dimension to be significantly larger than the Planck scale, one can try to relate the electroweak scale to the fundamental higher dimensional Planck scale [2, 3, 4]. Alternatively, it has been suggested that an exponential scaling of the metric along the extra dimension (or "warp" factor) can also lead to a resolution of the hierarchy problem [5]. In both of these approaches the extra dimension may be as large as a $\mathrm{mm}$ scale (in the former) and even infinite (in the latter, see also [6]). In these theories, it is necessary to assume that standard model particles are confined to a $3+1$-dimensional brane of an $n+3+1$-dimensional space-time. On the other hand, gravity propagates in the bulk.

Many theories have been constructed using the notion that there are in fact two branes which represent the boundaries of a higher dimensional space-time. Such was the motivation from the Horava-Witten model in M-theory [1]. Indeed, there has been considerable effort to produce cosmological models on such boundaries [7, 8, 9]. In a different set of solutions [5], the brane is a slice of AdS and the negative cosmological constant of the bulk is used to cancel the cosmological constant or tension on the brane. In the single brane scenario [6], gravity is effectively confined to the brane by the steep warp factor generated by the tension dominating the brane. In most other constructions, two branes are necessary to achieve compactification. Of course in realistic cosmologies, the energy density of the Universe must be dominated by matter.

An important observation about the cosmology of the "brane world" was made in Refs. [8, 9, 10]. These papers showed that the Hubble parameter $H$ governing the expansion of the scale factor on the brane has a different behavior than derived from the usual 4-d Friedmann equations. In particular, the Hubble parameter is proportional to the energy density on the brane instead of the familiar dependence $H \sim \sqrt{\rho}$. While mathematically correct as a solution to Einstein's equations, this behavior, if nothing else, indicates that 
some key ingredient is missing if this theory is to be capable of describing our Universe. It is worth noting that this unusual behavior of $H$ does not depend on the size of the extra dimension, but holds even for $r \sim M_{*}^{-1} \sim M_{\mathrm{Pl}}^{-1}$. While this abnormal behavior of $H$ may be possible at very early cosmological epochs which cannot be immediately confronted with observational data, the $H \sim \rho$-type of behavior is totally unacceptable at later times, particularly at big bang nucleosynthesis.

The origin of the aberrant expansion law can be traced to a condition, imposed on the solution of the 5D Einstein's equations, which relates the energy density on the two branes. Roughly speaking, the presence of matter on the 3-brane, produces a $y$-dependence $(y$ is the spatial coordinate of the extra dimension) of the 3-space scale factor $a$ such that $a(y)$ decreases as one moves away from the brane. Indeed for an infinitely thin brane, there is a discontinuity in the derivative of $a$ with respect to $y$. (This discontinuity is removed by considering branes of finite thickness, but the qualitative behavior of the solutions are unchanged as we will show below.) For a brane dominated by a positive tension, $a(y)$ decreases exponentially away from the brane, and in effect compactification is unnecessary [6]. If the brane is not dominated by the tension, the decrease in $a(y)$ is softer and compactification may be necessary. To compactify, a second brane must be placed at some fixed distance from the initial brane. The scale factor will also experience a discontinuity (in $a^{\prime}(y)$ ) on the second brane, but of opposite sign. This distinction imposes very severe constraints on the matter and tension of the second brane. In fact, if the energy density on the original brane is $\rho_{1}$, then on the second brane it must be $\rho_{2}=-\rho_{1}+O\left(\rho^{2}\right)$. At cosmological distance scales (much larger than the separation of the branes), the Hubble parameter will be given by $H^{2} M_{P}^{2}=\rho_{1}+\rho_{2} \sim \rho^{2}$. This point was recently emphasized in [11].

A number of "remedies" to this problem have been suggested [12, 13, 14, 15]. Most of these works exploit the Randall-Sundrum construction with two branes of opposite tensions $\pm \Lambda_{b r}$ and obtain the correct cosmological Friedmann equation by cancelling the leading $\Lambda_{b r}^{2}$ proportional term with a negative bulk cosmological constant $\Lambda_{B}$. The remaining cross-term leads to the normal expansion law and the 4D Plank scale is related to the tension on the brane. For an alternative solution to this problem see 16]. Cosmology of the single-brane scenario of ref. [6] has been considered in [13, 17]. For additional papers of interest related to the Randall-Sundrum scenario see [18].

In a different approach to the problem 19] we derived sufficient conditions which ensure a smooth transition to conventional cosmology and Newton's law on the brane. We found that if $T_{\mu \nu}^{b u l k} \neq 0$, and in particular, $T_{55} \neq 0$ on the brane and in the bulk, the transition to the conventional cosmology can be obtained (for both thin and thick branes). Furthermore, the same class of solutions [19] allowed for the possibility of compactification with single brane, and obviates the need for an exotic object such as a brane with negative matter density. 
The main result achieved in [19] is that the desired resulting expansion law $\left(H^{2} \propto \rho\right)$, together with a fixed size $\left(L=\right.$ const) for the extra dimension can be achieved when $T_{55} \neq 0$. In fact, the specific value of $T_{5}^{5}$ must be chosen to be proportional to the trace of the $4 \mathrm{D}$ energy-momentum tensor on the brane and inversely proportional to the size of an extra dimension, $T_{5}^{5} \propto(-\rho+3 p) / 2 L+O\left(\rho^{2}\right)$. It was hypothesized in [19 that this value is in fact the consequence of the physics responsible for the stabilization of the dilaton.

In this paper we take this idea one step further and study the relation between $T_{5}^{5}$ and the dilaton fixing in more detail. In the absence of the dilaton stabilization mechanism certain conditions should be imposed on the 5D energy-momentum tensor to preserve dilaton flatness and thus the stability of the radius of an extra dimension. Working to linear order in energy density (assumed small), we find a generic integral condition on the energy-momentum tensor of 5D matter. When the explicit dilaton potential is introduced, we show that $T_{5}^{5} \propto(-\rho+3 p) / 2 L+O\left(\rho^{2}\right)$ arises as a back-reaction of the dilaton potential in the presence of the brane and, to this order, does not depend on the details of the dilaton fixing potential. That is, the solution found in [19] is completely generic.

Another important goal that we pursue in this paper is the development of our initial solution [19] in several new directions. In particular, we study whether the thin wall solution with a compact extra dimension allows for the generalization on the case of small but finite thickness. We also extend our solution to include cosmological constants on the brane and in the bulk, without restricting the sign of the bulk cosmological constant. In all of these cases we recover normal Friedmann-type solutions on the brane by requiring the stability of the transverse dimension.

We organize this paper as follows. The main ingredients of the model, Einstein equations specialized for particular ansatzes for the metric and energy-momentum tensor, are introduced in the next section. Section 3 deals with the interpretation of the (55)-component of the energy-momentum tensor in the bulk. First, we consider the condition for dilaton flatness in the presence of $5 \mathrm{D}$ matter and show the desirable form of $T_{5}^{5}$ which satisfies this condition. Then we turn to the most important case when the dilaton receives an explicit potential and demonstrate how $T_{5}^{5}$ is generated as the response of this potential to the presence of the brane. In section 4, the thin wall solution is generalized to take into account cosmological constants on the brane and in the bulk. We address the cases of negative, positive and zero cosmological constant in the bulk. In section 5 the single brane solution with compactification is analyzed when the brane is given a certain thickness $\Delta \ll L$. Our conclusions are presented in section 6 . 


\section{The Theoretical Framework}

As an example of a higher-dimensional theory describing the coupling of the matter content of the universe with gravity, we consider the following 5-dimensional theory

$$
S=\int d^{5} x \sqrt{-G^{(5)}}\left\{\frac{M_{5}^{3}}{16 \pi} \hat{R}+\hat{\mathcal{L}}_{o}\right\}
$$

where $\hat{\mathcal{L}}_{o}$ represents all possible contributions to the action which are not strictly gravitational. In the above, $M_{5}$ is the fundamental 5-dimensional Planck mass, and the hat will denote 5-dimensional quantities. The line-element of the 5-dimensional manifold is given by the following ansatz

$$
d s^{2}=-n^{2}(t, y) d t^{2}+a^{2}(t, y) \delta_{i j} d x^{i} d x^{j}+b^{2}(t, y) d y^{2}
$$

where $\left\{t, x^{i}\right\}$ and $y$ denote the usual, 4-dimensional spacetime and the extra dimension, respectively.

When the action functional (2.1) is varied with respect to the 5-dimensional metric tensor $G_{M N}^{(5)}$, Einstein's equations are derived, which for the above spacetime background take the form (see e.g. [8, [10])

$$
\begin{aligned}
\hat{G}_{00} & =3\left\{\frac{\dot{a}}{a}\left(\frac{\dot{a}}{a}+\frac{\dot{b}}{b}\right)-\frac{n^{2}}{b^{2}}\left[\frac{a^{\prime \prime}}{a}+\frac{a^{\prime}}{a}\left(\frac{a^{\prime}}{a}-\frac{b^{\prime}}{b}\right)\right]\right\}=\hat{\kappa}^{2} \hat{T}_{00}, \\
\hat{G}_{i i} & =\frac{a^{2}}{b^{2}}\left\{\frac{a^{\prime}}{a}\left(\frac{a^{\prime}}{a}+2 \frac{n^{\prime}}{n}\right)-\frac{b^{\prime}}{b}\left(\frac{n^{\prime}}{n}+2 \frac{a^{\prime}}{a}\right)+2 \frac{a^{\prime \prime}}{a}+\frac{n^{\prime \prime}}{n}\right\} \\
& +\frac{a^{2}}{n^{2}}\left\{\frac{\dot{a}}{a}\left(-\frac{\dot{a}}{a}+2 \frac{\dot{n}}{n}\right)-2 \frac{\ddot{a}}{a}+\frac{\dot{b}}{b}\left(-2 \frac{\dot{a}}{a}+\frac{\dot{n}}{n}\right)-\frac{\ddot{b}}{b}\right\}=\hat{\kappa}^{2} \hat{T}_{i i}, \\
\hat{G}_{05} & =3\left(\frac{n^{\prime}}{n} \frac{\dot{a}}{a}+\frac{a^{\prime}}{a} \frac{\dot{b}}{b}-\frac{\dot{a}^{\prime}}{a}\right)=0, \\
\hat{G}_{55} & =3\left\{\frac{a^{\prime}}{a}\left(\frac{a^{\prime}}{a}+\frac{n^{\prime}}{n}\right)-\frac{b^{2}}{n^{2}}\left[\frac{\dot{a}}{a}\left(\frac{\dot{a}}{a}-\frac{\dot{n}}{n}\right)+\frac{\ddot{a}}{a}\right]\right\}=\hat{\kappa}^{2} \hat{T}_{55},
\end{aligned}
$$

where $\hat{\kappa}^{2}=8 \pi \hat{G}=8 \pi / M_{5}^{3}$ and the dots and primes denote differentiation with respect to $t$ and $y$, respectively.

The 5-dimensional energy-momentum tensor of the theory is obtained by the variation of $\sqrt{-G^{(5)}} \hat{\mathcal{L}}_{o}$ with respect to the metric and may be written in the form

$$
\hat{T}^{M}{ }_{N}=\operatorname{diag}\left(-\hat{\rho}, \hat{p}, \hat{p}, \hat{p}, \hat{T}_{5}^{5}\right)
$$


and, when combined with the equation for the conservation of the energy-momentum tensor, $D_{M} \hat{T}^{M}{ }_{N}=0$, leads to the following relations

$$
\begin{array}{r}
\frac{d \hat{\rho}}{d t}+3(\hat{\rho}+\hat{p}) \frac{\dot{a}}{a}+\left(\hat{\rho}+\hat{T}_{5}^{5}\right) \frac{\dot{b}}{b}=0, \\
\left(\hat{T}_{5}^{5}\right)^{\prime}+\hat{T}_{5}^{5}\left(\frac{n^{\prime}}{n}+3 \frac{a^{\prime}}{a}\right)+\frac{n^{\prime}}{n} \hat{\rho}-3 \frac{a^{\prime}}{a} \hat{p}=0 .
\end{array}
$$

In what follows, we adopt the brane-world scenario and, thus, we assume that the usual matter content of the universe is confined on a brane which may have zero or non-zero thickness. However, we make no such assumption regarding the fifth component $\hat{T}_{5}^{5}$ which can be smoothly distributed along the extra dimension while non-vanishing $\hat{\rho}$ and $\hat{p}$ may exist in the bulk due to a cosmological constant.

In the framework of the above theory, we are looking for cosmological solutions which admit compactification of the extra dimension and, at the same time, respect the usual form of the 4-dimensional Friedmann equation that arises from Einstein's equations in the limit $y \rightarrow 0$. Since we are interested in a compact extra dimension, we impose the condition that the scale factor of the fifth dimension depends neither on space nor time, i.e. $b=b_{0}=$ const. In that case, our task is greatly simplified since the (05)-component (2.5) of Einstein's equations can be easily integrated to give the result

$$
n(t, y)=\lambda(t) \dot{a}(t, y) .
$$

Moreover, the (00)-component (2.3), now, reduces to a second-order differential equation for $a(t, y)$ with respect to $y$ with the general solution depending on the form of the energy density $\hat{\rho}$ of the universe. The function $\lambda(t)$ is of equal importance as it leads, not only to the determination of the lapse function $n(t, y)$, but also to the 4-dimensional Friedmann equation at the origin, that is, on the brane. Using the normalization $n(t, y=0)=1$, the Hubble parameter can be expressed in terms of $\lambda(t)$ in the following way

$$
H^{2} \equiv\left(\frac{\dot{a}_{0}}{a_{0}}\right)^{2}=\frac{1}{\lambda^{2}(t) a_{0}^{2}(t)},
$$

where the subscript 0 denotes quantities evaluated at $y=0$. As it will be demonstrated shortly, the form of the function $\lambda(t)$, and thus the restoration of the Friedmann equation at the origin, will strongly depend on the expression of the fifth component of the energymomentum tensor $\hat{T}_{5}^{5}$.

It is important to remark at this point, that so far, we have considered the constancy of the scale factor $b$ as an external condition. That is, it is not one which is obtained automatically from the solution of Einstein's equations. Fixing $b$, is equivalent to fixing 
the vev of the dilaton in the dimensionally reduced theory. This is clearly a physically reasonable assumption since a rolling dilaton implies the variation of gauge couplings and particle masses and there are very strong constraints against this 20. Indeed one would expect that a dilaton potential is generated elsewhere in the theory (buried in $\hat{\mathcal{L}}_{o}$ ) and provides for the necessary additional condition which fixes $b$. As we shall see in the next section, such a potential automatically gives rise to the $T_{55}$ component advocated in 19 .

\section{Stabilization of the extra dimension}

Suppose for a moment that we consider the simple example of a 5D Kaluza-Klein theory without matter. As such, we will have a solution for any size of the compactified dimension, i.e. the dilaton potential is flat. Let us now suppose that 5D matter with a given energymomentum tensor is distributed in this space. Clearly, there is no guarantee that a constant radius will remain an integral of motion unless the energy-momentum of the matter satisfies certain constraints. We next consider the conditions which must be imposed on $\hat{T}_{N}^{M}$ in order to preserve the dilaton flatness.

In some sense the answer to this question is similar to the following toy example. Imagine a massless scalar field $\phi(x)$ in $d$ dimensions which interacts with an external current $J(x)$. The condition

$$
\int J(x) d^{d} x=0
$$

ensures the stability of any vacuum configuration $\langle\phi(x)\rangle=v$ at the classical level. An analogous integral condition exists in the case of the Kaluza-Klein theory. The dilaton remains in equilibrium and the size of an extra dimension stays fixed if the stress energy of the 5D matter satisfies the following constraintt:

$$
\int d^{4} x d y \sqrt{-G^{(5)}}\left(\hat{T}_{\mu}^{\mu}-2 \hat{T}_{5}^{5}\right)=0
$$

To show how this condition actually emerges we work out an example of $\hat{T}_{N}^{M}$ created by a 5D scalar field, noting that the same arguments can be extended for other types of matter. We start by making a standard Kaluza-Klein decomposition of our diagonal metric (2.2) in the following way

$$
G_{M N}^{(5)}=\left(\begin{array}{cc}
G_{\mu \nu}^{(4)} & 0 \\
0 & e^{2 \gamma}
\end{array}\right)
$$

where $G_{\mu \nu}^{(4)}$ denotes the usual 4-dimensional metric and $e^{2 \gamma(t)} \equiv b^{2}(t)$. Here, for the sake of our analysis, we restore the time-dependence of the scale factor along the extra dimension.

\footnotetext{
${ }^{1}$ This constraint was also derived from a topological argument in [21].
} 
We also consider the following 5 -dimensional field theory of a single scalar field minimally coupled to gravity

$$
S=-\int d^{4} x d y \sqrt{-G^{(5)}}\left\{\frac{R^{(5)}}{2 \hat{\kappa}^{2}}+\frac{1}{2} \partial_{M} \phi \partial^{M} \phi+V(\phi)\right\} .
$$

where $\hat{\kappa}^{2}=8 \pi / M_{5}^{3}$. Under the aforementioned decomposition (3.3), the above theory can be written as

$$
S=-\int d^{4} x d y \sqrt{-G^{(4)}} e^{\gamma}\left\{\frac{1}{2 \hat{\kappa}^{2}}\left[R^{(4)}(t, y)-2 e^{-\gamma} D_{\mu} D^{\mu} e^{\gamma}\right]+\frac{1}{2} \partial_{M} \phi \partial^{M} \phi+V(\phi)\right\}
$$

The above form of the theory is not particularly convenient for our analysis: the "kinetic" term for the dilaton field $\gamma$ is a total derivative, and, thus, drops out from the action and, moreover, there is a direct coupling between $\gamma$ and $R^{(4)}(t, y)$. Both of these problems can be overcome by making the conformal transformation $G_{\mu \nu}^{(4)}=e^{-\gamma} g_{\mu \nu}$. Then, the action functional of the theory takes the final form

$$
S=-\int d^{4} x d y \sqrt{-g}\left\{\frac{1}{2 \hat{\kappa}^{2}}\left[R^{(4)}(t, y)+\frac{3}{2} \partial_{\mu} \gamma \partial^{\mu} \gamma\right]+\frac{1}{2} \partial_{\mu} \phi \partial^{\mu} \phi+\frac{1}{2} e^{-3 \gamma}\left(\partial_{5} \phi\right)^{2}+e^{-\gamma} V(\phi)\right\} .
$$

Our next task is to derive the equation of motion for the dilaton field $\gamma$ and find the condition that the components of the energy-momentum tensor of matter need to satisfy in order to ensure the stability of the extra dimension. The variation of the action (3.6) with respect to $\gamma$ is given by the expression

$$
\delta S=\int d y \sqrt{-g}\left(\frac{3}{2 \hat{\kappa}^{2}} D_{\mu} D^{\mu} \gamma-\left[-\frac{3}{2} e^{-3 \gamma}\left(\partial_{5} \phi\right)^{2}-e^{-\gamma} V(\phi)\right]\right) \delta \gamma,
$$

from which we learn that the stability condition, analogous to (3.1) is

$$
\int d y \sqrt{-g}\left[\frac{3}{2} e^{-3 \gamma}\left(\partial_{5} \phi\right)^{2}+e^{-\gamma} V(\phi)\right]=0
$$

This constraint can be expressed in terms of the components of the energy-momentum tensor generated by the 5-dimensional scalar field. Before making the conformal transformation of the 4-dimensional metric, $\hat{T}_{M N}$ had the form

$$
\hat{T}_{M N}=\partial_{M} \phi \partial_{N} \phi-G_{M N}^{(5)}\left[\frac{1}{2} \partial_{P} \phi \partial^{P} \phi+V(\phi)\right] .
$$

Then, we can easily find the following components

$$
\begin{aligned}
& \hat{T}_{\mu}^{\mu}=G^{(4) \mu \nu} \hat{T}_{\mu \nu}=-\partial_{\mu} \phi \partial^{\mu} \phi-2 e^{-2 \gamma}\left(\partial_{5} \phi\right)^{2}-4 V(\phi), \\
& \hat{T}_{5}^{5}=e^{-2 \gamma} \hat{T}_{55}=-\frac{1}{2} \partial_{\mu} \phi \partial^{\mu} \phi+\frac{1}{2} e^{-2 \gamma}\left(\partial_{5} \phi\right)^{2}-V(\phi) .
\end{aligned}
$$


We can now rewrite the constraint equation (3.8) preventing a linear source for the dilaton $\gamma$ in terms of $\hat{T}_{M N}$ which up to a multiplicative constant yields

$$
\int d y \sqrt{-g} \frac{e^{-\gamma}}{2}\left(\hat{T}_{\mu}^{\mu}-2 \hat{T}_{5}^{5}\right)=0 .
$$

Note that, although $\hat{T}_{\mu}^{\mu}$ and $\hat{T}_{5}^{5}$ are not conformally invariant, their combination $\hat{T}_{\mu}^{\mu}-2 \hat{T}_{5}^{5}$ is, since the difference does not depend explicitly on $G_{\mu \nu}^{(4)}$. Therefore, the above result remains invariant under the conformal transformation that we impose on the 4-dimensional part of the metric tensor.

We can easily demonstrate that the introduction of any type of matter in the context of the 5D theory serves as a source term for the $4 \mathrm{D}$ gravitons. More specifically, consider the linear term

$$
\delta G_{M N}^{(5)} \hat{T}^{M N}=\delta G_{\mu \nu}^{(4)} \hat{T}^{\mu \nu}+\delta\left(e^{2 \gamma}\right) \hat{T}^{55}
$$

in any system. Using $G_{\mu \nu}^{(4)}=e^{-\gamma} g_{\mu \nu}$, one can rewrite the above expression as

$$
\delta G_{M N}^{(5)} \hat{T}^{M N}=e^{-\gamma} \delta g_{\mu \nu} \hat{T}^{\mu \nu}-\delta \gamma\left(\hat{T}_{\mu}^{\mu}-2 \hat{T}_{5}^{5}\right)
$$

Thus, we see that $\hat{T}_{\mu \nu}$ is a source for the graviton while the combination $\left(\hat{T}_{\mu}^{\mu}-2 \hat{T}_{5}^{5}\right)$ is the source for the dilaton or for any Brans-Dicke scalar field. In the presence of a mechanism that stabilizes the extra dimension, the source for the dilaton is identically zero. If we do not have a stabilization mechanism but, nevertheless, we look for solutions with a Newtonian limit, we still have to require the absence of the above term. On the other hand, the presence of a scalar field in the theory will give rise, not to a standard Einstein theory of gravity, but to a scalar-tensor theory with all its consequences.

All of these considerations help to elucidate the required form for $\hat{T}_{N}^{M}$ in the 5-dimensional space which allows us to keep the size of the extra dimension fixed. Eq. (3.2) is the constraint which in fact must be satisfied. This constraint can, for example, be easily specialized to the cases already considered in previous publications. Following [10], one may choose two branes with opposite matter densities and vanishing $\hat{T}_{5}^{5}$. In this case the stability condition (3.2) is trivially satisfied (since the $\hat{T}_{\mu}^{\mu}$ 's of each brane cancel each other). As another illuminating example, we consider the thin-wall cosmological solution that was derived in Ref. [19] and describes the 5-dimensional space-time around an infinitely thin brane-universe located at $y=0$. The ordinary matter density is confined to the brane and no other form of energy - apart from a smooth distribution of $\hat{T}_{5}^{5}$ - exists in the bulk. The energy-momentum tensor of the brane-universe, in this case, has the form

$$
\hat{T}_{N}^{M}=\operatorname{diag}\left(\frac{\delta(y)}{b_{0}}(-\rho, p, p, p), \hat{T}_{5}^{5}\right) .
$$

The expression for $\hat{T}_{5}^{5}$ in the bulk follows from the assumption that stable extrema of $n(y)$ and $a(y)$ exist outside the brane, at $y= \pm\left|y_{\min }\right|$, and that the Friedmann equation has the 
usual form at the origin. The resulting energy-momentum tensor component is

$$
\hat{T}_{5}^{5}=\frac{a_{0}^{3}(t)}{2 n(t, y) a^{3}(t, y)} \frac{(-\rho+3 p)}{2 b_{0}\left|y_{\min }\right|}+\mathcal{O}\left(\rho^{2}\right) .
$$

Note that, in our expansion in powers of $\rho$, the prefactor $\left(a_{0}^{3} / n a^{3}\right)=1+\mathcal{O}\left(\rho^{2}\right)$, so that $\hat{T}_{5}^{5}=(-\rho+3 p) /\left(2 b_{0}\left|y_{\min }\right|\right)+\mathcal{O}\left(\rho^{2}\right)$.

Going back to eq. (3.15), the 4-dimensional trace of the energy-momentum tensor is simply given by the expression

$$
\hat{T}_{\mu}^{\mu}=\frac{\delta(y)}{b_{0}}(-\rho+3 p) .
$$

Taking into account the fact that the 4-dimensional part of the energy-momentum tensor is localized on the brane while $\hat{T}_{5}^{5}$ is not and performing the integration in (3.12) with respect to $y \in\left(-\left|y_{\min }\right|,\left|y_{\min }\right|\right)$, we can easily demonstrate that the desirable form of $\hat{T}_{5}^{5}$ satisfies our general constraint (3.2).

The general condition on the stability of the radius (3.2) represents a highly nontrivial fine-tuning problem for the matter fields in 5D space if one insists on a flat potential for the dilaton and on the compactification at an arbitrary value of $\gamma$. Moreover, the dilaton in this scenario will remain massless, which constitutes another serious phenomenological problem as noted above. Therefore, we must consider a more realistic situation with an additional physical input, namely an explicit mechanism which gives the mass to the dilaton and stabilizes it around a specific local minimum.

It is tantalizing to write this potential in the simplest possible form,

$$
V\left(G_{55}^{(5)}\right)=\alpha\left(G_{55}^{(5)}-b_{0}^{2}\right)^{2},
$$

so that $G_{55}^{(5)}=e^{2 \gamma}=b_{0}^{2}$ represents an equilibrium point. Since this form explicitly breaks general covariance, it must be only effective, realized by some bulk mechanism, unspecified at this point. Any uniform deviation of $G_{55}^{(5)}$ from $b_{0}^{2}$ induces a uniform value for $\hat{T}_{5}^{5}$ in the bulk, given by the derivative of $V$ with respect to $G_{55}^{(5)}$. If a brane with the nonvanishing matter energy density is included, the equilibrium position of $G_{55}^{(5)}$ changes. We next show that the value of $\hat{T}_{5}^{5}$, generated this way, does satisfy eq. (3.16) independent of the parameters which characterize the stiffness of the dilaton potential.

To have a workable framework which preserves general covariance we choose an auxiliary bulk scalar $\chi$ with the following unusual action

$$
S=-\int d^{4} x \int_{-\left|y_{\min }\right|}^{\left|y_{\min }\right|} \sqrt{-G^{(5)}} d y\left(\partial_{M} \chi \partial^{M} \chi-c_{1}^{2}\right)^{2}
$$

in the given background configuration $\chi(y)=c_{2}|y|$. This background configuration creates the potential of the form (3.18) if we identify $b_{0}=c_{2} / c_{1}$. The dilaton chooses its preferred 
point $\gamma_{0}$ and it is easy to check that $G_{55}^{(5)}=e^{2 \gamma_{0}}=b_{0}^{2}=c_{2}^{2} / c_{1}^{2}$ together with $\chi(y)=c_{2}|y|$ satisfy all of the equations of motions. At the same time, at the equilibrium position, all the components of $\hat{T}_{N}^{M}$ which correspond to the action (3.19) remain identically zero. We also note here that such a background configuration for $\chi$ is consistent with compactification and it can be further checked that with the choice of (3.19), the cusps in $\chi(y)$ do not require the existence of delta-functional sources.

When a brane with matter content is introduced at $y=0$, the equilibrium position for $\gamma$ changes. This change can be read off from the interaction of $\gamma$ with the trace of the brane energy-momentum tensor, eq. (3.12), and the effective potential generated by (3.19). Keeping only the term linear in this deviation, $\gamma-\gamma_{0}$, in front of the small perturbation created by $\hat{T}_{\mu}^{\mu}$ of the brane, we write down an effective potential for the dilaton of the form

$$
V_{e f f}(\gamma)=\frac{8}{e^{5 \gamma_{0}}}\left|y_{\text {min }}\right| c_{2}^{4}\left(\gamma-\gamma_{0}\right)^{2}-\frac{1}{2 e^{2 \gamma_{0}}}(-\rho+3 p)\left(\gamma-\gamma_{0}\right)
$$

from which we see that the new equilibrium point is given by

$$
\left(\gamma-\gamma_{0}\right)=\frac{e^{3 \gamma_{0}}}{c_{2}^{4}} \frac{(-\rho+3 p)}{32\left|y_{\text {min }}\right|} .
$$

This deviation produces a non-vanishing (55)-component of the energy-momentum tensor in the bulk (from the variation of 3.19 ), exactly of the form discussed above. Indeed, neglecting all terms on the order of $\rho^{2}$, we have the following value for $\hat{T}_{5}^{5}$

$$
\hat{T}_{5}^{5}=8 c_{2}^{4} e^{-4 \gamma_{0}}\left(\gamma-\gamma_{0}\right)=\frac{-\rho+3 p}{4 b_{0}\left|y_{\min }\right|},
$$

whereas the (00) and $(i i)$ components of $\hat{T}_{N}^{M}$ in the bulk remain zero to this accuracy. The same arguments hold for any mechanism which would render an effective potential in the form (3.18).

Equation (3.22), is exactly of the same value needed for the restoration of the Friedmann equation for the brane scale factor, and allows for an important interpretation of $\hat{T}_{5}^{5}$. Indeed, we saw that this component arises as the backreaction of the dilaton fixing potential on the presence of the energy density on the brane. This confirms a hypothesis put forward in our previous work [19]. At the same time it removes the necessity of the fine tuning (3.12) required for the case of the flat potential. It turns out that the only requirements which should be imposed on $\alpha$ in (3.18) or $c_{2}^{2}$ in (3.19)-(3.22) is the condition $\gamma-\gamma_{0} \ll \gamma_{0}$ which justifies our linearized approach. We see that the details of the dilaton fixing potential are irrelevant to linear order in $\rho$ and affect only the sub-leading, $\rho^{2}$-proportional terms. We note that mechanisms for generating a dilaton potential from bulk fields was discussed in [22]. 
Recent work on the stabilization of the dilaton in the context of the Randall-Sundrum model showed a similar shift in the dilaton expectation value [23]. Indeed, the shift found there and the resulting change in the derivative of the dilaton potential at the shifted point acts as a source to $\hat{G}_{55}$ giving an identical expression for $\hat{T}_{55}$ described here and in [19].

\section{Solutions with cosmological constant and compact- ification}

In this section, we adopt the scenario of an infinitely thin brane-universe located at $y=0$ with the ordinary energy density and pressure being localized on the brane. We further assume that the total energy density of the brane, $\rho_{b r}$, is the sum of the ordinary matter density, $\rho$, and a brane cosmological constant, $\Lambda_{b r}$. On the other hand, $\hat{T}_{5}^{5}$ is smoothly distributed along the extra dimension while the bulk is dominated by the energy density coming from a bulk cosmological constant, $\Lambda_{B}$. Here, we are going to investigate the existence of cosmological solutions that admit compactification of the extra dimension while leading, once again, to the standard cosmological expansion of the brane scale factor. We will consider three different cases corresponding to negative, positive and zero bulk cosmological constant. The sign of $\Lambda_{b r}$ will be determined by demanding the cancellation of the effective cosmological constant in the Friedmann equation. Related brane solutions with both brane and bulk cosmological constant were considered in [12, 24].

\subsection{Negative cosmological constant in the bulk}

We start with the case of a negative bulk cosmological constant, $\Lambda_{B}<0$, and we write the energy-momentum tensor in the bulk in the following form

$$
\hat{T}_{B}^{A}=\left(-\rho_{B}, p_{B}, p_{B}, p_{B}, \hat{T}_{5}^{5}\right),
$$

where $\rho_{B}=\Lambda_{B}<0$ and the other components are, for the moment, arbitrary. For a smooth, constant distribution of energy in the bulk, the solution for the scale factor $a(t, y)$, outside the brane, will follow from the (00)-component of Einstein's equations, which takes the simple form

$$
x^{\prime \prime}-A^{2} x-B^{2}=0,
$$

where $x \equiv a^{2}$ and

$$
A^{2}=\frac{2 \hat{\kappa}^{2}}{3} b^{2}\left|\Lambda_{B}\right|, \quad B^{2}(t)=\frac{2 b^{2}}{\lambda^{2}(t)} .
$$

The general solution of the above differential equation has the form

$$
a^{2}(t, y)=d_{1}(t) \cosh (A|y|)+d_{2}(t) \sinh (A|y|)-\frac{B^{2}(t)}{A^{2}} .
$$


The boundary conditions on the brane will determine the two unknown functions of time, $d_{1}(t)$ and $d_{2}(t)$. Defining $a^{2}(t, y=0) \equiv a_{0}^{2}(t)$, we can write

$$
d_{1}(t)=a_{0}^{2}(t)+\frac{B^{2}(t)}{A^{2}}
$$

while the jump in the first derivative of $a(t, y)$ across the origin, which now takes the form

$$
\frac{\left[a^{\prime}\right]}{a_{0} b}=-\frac{\hat{\kappa}^{2}}{3}\left(\rho+\Lambda_{b r}\right)
$$

leads to the following expression for the second function of time

$$
d_{2}(t)=-\frac{\hat{\kappa}^{2}}{3} \frac{a_{0}^{2} b}{A}\left(\rho+\Lambda_{b r}\right)
$$

When the general solution (4.4) is substituted in the (ii)-component of Einstein's equations, we get the anticipated result $p_{B}=\left|\Lambda_{B}\right|=-\rho_{B}$ while, the same procedure with the (55)-component, leads to the constraint

$$
\frac{d}{d t}\left[\frac{A^{2}}{4}\left(d_{2}^{2}-d_{1}^{2}\right)+\frac{b^{4}}{A^{2} \lambda^{4}}\right]=\frac{2 \hat{\kappa}^{2}}{3} b^{2} a^{3} \dot{a}\left(\hat{T}_{5}^{5}-\left|\Lambda_{B}\right|\right) .
$$

The above constraint will allow us to determine the form of $\hat{T}_{5}^{5}$ once the expression for $\lambda(t)$ is fixed by the Friedmann equation.

Next, we come to the question of the compactification. Even in the absence of a second brane, the compactification of the extra dimension could easily take place as long as the solution (4.4) has a stable extremum at some point $y=\left|y_{\min }\right|$. Then, by identifying the points $y= \pm\left|y_{\min }\right|$, the extra dimension would be compactified with its size being $2 b\left|y_{\min }\right|$. From the vanishing of $a^{\prime}(t, y)$, we find that

$$
\tanh \left(A\left|y_{\min }\right|\right)=-\frac{d_{2}(t)}{d_{1}(t)} \equiv c_{0} .
$$

The above ratio, as indicated, must be constant in order for the extremum to be stable in time. Then, by using the above relation and eqs. (4.5) and (4.7), the Friedmann equation immediately follows

$$
\left(\frac{\dot{a}_{0}}{a_{0}}\right)^{2}=\frac{1}{\lambda^{2} a_{0}^{2}}=\frac{\hat{\kappa}^{2}}{3}\left|\Lambda_{B}\right|\left\{-1+\frac{\hat{\kappa}^{2}}{c_{0}} \frac{\left(\rho+\Lambda_{b r}\right)}{\sqrt{6 \hat{\kappa}^{2}\left|\Lambda_{B}\right|}}\right\} \equiv \frac{\kappa^{2} \rho}{3} .
$$

The last equality in the above equation defines the 4D Planck constant and holds only under the constraint

$$
-1+\frac{\hat{\kappa}^{2}}{c_{0}} \frac{\Lambda_{b r}}{\sqrt{6 \hat{\kappa}^{2}\left|\Lambda_{B}\right|}}=0
$$


and the redefinition

$$
\kappa^{2}=\frac{\hat{\kappa}^{2}}{c_{0}} \sqrt{\frac{\hat{\kappa}^{2}\left|\Lambda_{B}\right|}{6}}
$$

These expressions can also be used to define the 4D cosmological constant on the brane. From eq. (4.10), we can write

$$
\begin{aligned}
\Lambda_{e f f} & =\frac{\hat{\kappa}^{2}}{\kappa^{2}}\left|\Lambda_{B}\right|\left\{-1+\frac{\hat{\kappa}^{2}}{c_{0}} \frac{\Lambda_{b r}}{\sqrt{6 \hat{\kappa}^{2}\left|\Lambda_{B}\right|}}\right\} \\
& =\Lambda_{b r}-c_{0} \sqrt{\frac{6\left|\Lambda_{B}\right|}{\hat{\kappa}^{2}}}
\end{aligned}
$$

In the limit that $A\left|y_{\text {min }}\right|$ is large, $c_{0} \simeq 1$ and $\Lambda_{\text {eff }}=\Lambda_{b r}-\sqrt{\frac{6\left|\Lambda_{B}\right|}{\hat{\kappa}^{2}}}$. In the other limiting case, when $A\left|y_{\text {min }}\right|$ is small, we have $\Lambda_{e f f}=\Lambda_{b r}-\left(2 b\left|y_{\text {min }}\right|\right) \Lambda_{B}$.

From its definition (4.9), since $A\left|y_{\text {min }}\right|$ is positive, it is easy to see that $c_{0}$ is always positive independently of the exact values of $\left|\Lambda_{B}\right|$ and $\left|y_{\text {min }}\right|$ and, thus, the Friedmann equation will always have the correct sign. Then, in order for the constraint (4.11) to be satisfied, we must necessarily have $\Lambda_{b r}>0$. The above two equations can be rewritten in a simple form as

$$
\left|\Lambda_{B}\right|=\frac{\hat{\kappa}^{2}}{6 c_{0}^{2}} \Lambda_{b r}^{2}, \quad \kappa^{2}=\frac{\hat{\kappa}^{4}}{6 c_{0}^{2}} \Lambda_{b r}
$$

Finally, we need to determine the form of $\hat{T}_{5}^{5}$ in the bulk. By using the constraint (4.8) and the relations (4.14), we find that

$$
\hat{T}_{5}^{5}=\left|\Lambda_{B}\right|+\frac{\hat{\kappa}^{2} a_{0}^{3}}{12 n(t, y) a^{3}(t, y)}\left\{-\rho(\rho+3 p)+\left(1-\frac{1}{c_{0}^{2}}\right) \Lambda_{b r}\left(2 \Lambda_{b r}+\rho-3 p\right)\right\}
$$

A number of comments should be made at this point. In the limit $\left|y_{\min }\right| \rightarrow \infty$, the extra dimension is non-compact and $c_{0}=1$. In that case, the last term in the above expression becomes zero and, modulo a small correction of $\mathcal{O}\left(\rho^{2}\right), \hat{T}_{5}^{5}$ assumes the form of another pressure-like component of the energy-momentum tensor, equal to $\left|\Lambda_{B}\right|$. This is the case considered in Ref. [24]. In order to compactify, their solution requires the introduction of a second brane along with an unavoidable correlation between the energy densities on the two branes. In particular, the absence of $\hat{T}_{5}^{5}$, again implies the presence of a negative energy brane. For every other value of $c_{0}$, our results describe 5-dimensional cosmological solutions that respect the 4-dimensional Friedmann equation and allow for the compactification of the fifth dimension via the existence of an extremum of $a(t, y)$ at some finite point $y=\left|y_{\text {min }}\right|$. As we have seen in the previous section, the deviation of the fifth component of the energymomentum tensor from the strict limits of a pressure-like component, is crucial for the stabilization of the extra dimension. 
Let us, finally, note that the compactification of the extra dimension via the extremum of the scale factor not only eliminates the need for the introduction of a second brane but also resolves a major problem that arises in the framework of the two-brane models. For $\Lambda_{B}<0$, it has been shown [13, 14] that, in order to reproduce the correct sign in the Friedmann equation, one of the two branes must have an energy density which is negative, $\rho<0$. The need for this unnatural assumption ceases to exist in the context of our analysis. Despite the fact that we start with the same assumption of a negative cosmological constant in the bulk, in our case, the correct sign in the Friedmann equation is always maintained. Two- and multi-brane solutions in this context will be presented elsewhere.

\subsection{Positive cosmological constant in the bulk}

In this case, we assume that $\rho_{B}=\Lambda_{B}>0$. The analysis closely follows the one of the previous subsection, however, the opposite sign of $\Lambda_{B}$ modifies some of our results and, consequently, our final conclusions. We start again with the general solution for the scale factor $a(t, y)$ outside the brane, which, now, has the form

$$
a^{2}(t, y)=d_{1}(t) \cos (A|y|)+d_{2}(t) \sin (A|y|)+\frac{B^{2}}{A^{2}}
$$

The first coefficient $d_{1}(t)$ is given by eq. (4.5) with $A^{2}$ being replaced by $-A^{2}$ while the second one, $d_{2}(t)$, is still given by eq. (4.7). Once again, it is easy to see that the above solution satisfies the $(i i)$-component of Einstein's equations provided that $p_{B}=-\Lambda_{B}=-\rho_{B}$ while, the (55)-component, leads to the constraint

$$
\frac{d}{d t}\left[\frac{A^{2}}{4}\left(d_{2}^{2}+d_{1}^{2}\right)-\frac{b^{4}}{A^{2} \lambda^{4}}\right]=\frac{2 \hat{\kappa}^{2}}{3} b^{2} a^{3} \dot{a}\left(\hat{T}_{5}^{5}+\Lambda_{B}\right) .
$$

We still need a stable extremum for the compactification of the extra dimension and this extremum, now, takes place at

$$
\tan \left(A\left|y_{\min }\right|\right)=\frac{d_{2}(t)}{d_{1}(t)} \equiv c_{0}
$$

By using the above relation and the expressions for $d_{i}(t)$, the Friedmann equation can, now, be written as

$$
\left(\frac{\dot{a}_{0}}{a_{0}}\right)^{2}=\frac{\hat{\kappa}^{2}}{3} \Lambda_{B}\left\{1+\frac{\hat{\kappa}^{2}}{c_{0}} \frac{\left(\rho+\Lambda_{b r}\right)}{\sqrt{6 \hat{\kappa}^{2} \Lambda_{B}}}\right\} .
$$

The standard form of the above equation is immediately restored under the constraint

$$
1+\frac{\hat{\kappa}^{2}}{c_{0}} \frac{\Lambda_{b r}}{\sqrt{6 \hat{\kappa}^{2} \Lambda_{B}}}=0
$$


while the redefinition of the 4-dimensional Newton's constant in terms of the 5-dimensional one is still given by eq. (4.12). From its definition, eq. (4.18), we conclude that $c_{0}$ is not always positive. Thus, in order to have the correct sign in the Friedmann equation, we have to impose the following constraint on the possible values of $\left|y_{\min }\right|$

$$
n \pi<A\left|y_{\min }\right|<\left(n+\frac{1}{2}\right) \pi, \quad n \in Z .
$$

Then, the constraint (4.20) can be satisfied only in the case where $\Lambda_{b r}<0$. Note that the cosmological constants in the bulk and on the brane must always have opposite signs in order to ensure the cancellation of their contributions to the energy density of the universe. However, their sign does not enter the Friedmann equation neither imposes any unnatural constraints on the energy density of our brane-universe.

Let us note that, contrary to the case where $\Lambda_{B}<0$, here, there are no solutions that would correspond to a non-compact extra dimension. Every cosmological solution, described by eqs. (4.16) and (4.18), that respects the Friedmann equation at the origin has a compact extra dimension with finite size $2 b\left|y_{\text {min }}\right|$. This is also obvious from the expression for the fifth component of the energy-momentum tensor in the bulk, which from the constraint (4.17) is found to be

$$
\hat{T}_{5}^{5}=-\Lambda_{B}+\frac{\hat{\kappa}^{2} a_{0}^{3}}{12 n(t, y) a^{3}(t, y)}\left\{-\rho(\rho+3 p)+\left(1+\frac{1}{c_{0}^{2}}\right) \Lambda_{b r}\left(2 \Lambda_{b r}+\rho-3 p\right)\right\} .
$$

Note that the form of $\hat{T}_{5}^{5}$ is always different from the one of a pressure-like component and there is no real value of $c_{0}$ that would eliminate the last term. Thus, in order to stabilize the extra, compact dimension, a non-trivial $\hat{T}_{5}^{5}$ must be generated.

\subsection{Zero cosmological constant in the bulk}

Finally, we consider the case where $\Lambda_{B}=0$ while keeping a non-zero cosmological constant on the brane. In the bulk, the solution is exactly the same as in the case studied in the thin-wall approximation of our previous work [19]. The general solution for $a(t, y)$ can be written as

$$
a^{2}(t, y)=a_{0}^{2}(t)+c(t)|y|+\frac{b^{2}}{\lambda^{2}} y^{2}
$$

with

$$
c(t)=-\frac{\hat{\kappa}^{2}}{3} a_{0}^{2} b\left(\rho+\Lambda_{b r}\right)
$$

For $\rho+\Lambda_{b r}>0$, the above solution describes a spatial scale factor that decreases as we move away from the brane. However, the rate of decrease is much smaller than the one given by the warp factor in the Randall-Sundrum model [5]. 
The compactification of the extra dimension is made by identifying the two extrema which, now, take place at the value

$$
\left|y_{\min }\right|=-\frac{c(t) \lambda^{2}(t)}{2 b^{2}}
$$

and, by using the above result for $c(t)$, the Friedmann equation may be written as

$$
\left(\frac{\dot{a}_{0}}{a_{0}}\right)^{2}=\frac{\hat{\kappa}^{2}}{3} \frac{\left(\rho+\Lambda_{b r}\right)}{2 b\left|y_{\min }\right|}=\frac{\kappa^{2}}{3}\left(\rho+\Lambda_{b r}\right) .
$$

Due to the absence of a bulk cosmological constant that would cancel the contribution of $\Lambda_{b r}$ to the total energy of the universe, the term linear in $\rho$ will become dominant only in the case $\Lambda_{b r} \ll \rho$. In the opposite case, as anticipated, the presence of $\Lambda_{b r}$ will significantly modify the expansion rate of the scale factor at the origin.

\section{Thick-wall solution with compactification}

To complete the class of solutions we have been discussing, we generalize our thin-wall solution found in [19] to one of finite thickness. We assume that our brane-universe has a non-zero thickness $2 \Delta b$ with the normal matter content being distributed throughout the brane. Outside the brane, both the energy density and pressure are zero, which leads, after integrating the (00)-component of Einstein's equations, to the following general solution for the spatial scale factor

$$
a^{2}(t, y)=a_{\Delta}^{2}(t)+C(t)(|y|-\Delta)+\frac{b^{2}}{\lambda^{2}}\left(y^{2}-\Delta^{2}\right)
$$

where $a_{\Delta}(t)$ is the scale factor evaluated at $y=\Delta$ and $C(t)$ an unknown function of time which needs to be determined. For the compactification of the extra dimension, we require an extremum in $a(t, y)$ outside the brane, so, we impose the condition $a^{\prime}\left(t, y_{\text {min }}\right)=0$ on the general solution (5.1). That leads to the result

$$
\left|y_{\min }\right|=-\frac{C(t) \lambda^{2}}{2 b^{2}}
$$

In Ref. [19], we showed that, in the thin-wall approximation, the existence of a stable minimum for $a(t, y)$ outside the brane and, thus, the compactification of the extra dimension is equivalent to the restoration of the Friedmann equation at the origin. Here, we need to impose that this equation takes the usual form, i.e.

$$
\left(\frac{\dot{a}_{0}}{a_{0}}\right)^{2}=\frac{1}{\lambda^{2} a_{0}^{2}}=\frac{\kappa^{2} \rho(t, 0)}{3}
$$


where $\rho(t, 0)$ is the usual 4-dimensional energy density defined at the origin, and

$$
\kappa^{2}=\frac{\hat{\kappa}^{2}}{2 b\left|y_{\min }\right|}
$$

Then, by combining eqs. (5.2) and (5.3), we obtain

$$
C(t)=-\frac{\hat{\kappa}^{2} \rho(t, 0) a_{0}^{2} b}{3}
$$

It is easy to see that the solution (5.1) trivially satisfies the ( $i i)$-component of Einstein's equations while, when substituted in the (55)-component, it leads to the constraint

$$
\frac{d}{d t}\left[\frac{b^{2}}{\lambda^{4}}\left(\Delta-\left|y_{\min }\right|\right)^{2}-\frac{a_{\Delta}^{2}}{\lambda^{2}}\right]=\frac{2 \hat{\kappa}^{2}}{3} a^{3} \dot{a} \hat{T}_{5}^{5}
$$

The above constraint, with the expression for $\lambda$ given by eq. (5.3), is satisfied only if the fifth component of the energy-momentum tensor, in the bulk, has the form

$$
\hat{T}_{5(B)}^{5}(t, y)=\frac{1}{2 n a^{3}}\left\{\frac{a_{\Delta} a_{0}}{2 b\left|y_{\min }\right|}\left[a_{\Delta}(\rho+3 p)-2 \rho a_{0} n_{\Delta}\right]-\frac{\hat{\kappa}^{2} a_{0}^{3}}{6} \frac{\left(\Delta-\left|y_{\min }\right|\right)^{2}}{y_{\min }^{2}} \rho(\rho+3 p)\right\}
$$

where again $\rho$ and $p$ are defined at the origin. In the limit $\Delta \rightarrow 0$, the above result reduces to what we had for the thin-wall solution [19]. We emphasize once more, that despite the peculiar nature of the expression in (5.7), so long as the dilaton picks up a stable expectation value, this is the form that $T_{55}$ will assume.

To complete the thick wall solution, we must derive the expression for $\hat{T}_{5}^{5}(t, y)$ inside the brane. This derivation is given in the Appendix, the result is

$$
\hat{T}_{5(b r)}^{5}(t, y)=\hat{T}_{5(B)}^{5}(t, y)+\hat{\rho}\left[\frac{n(t, \Delta)}{n(t, y)}-1\right],
$$

where $\hat{T}_{5(B)}^{5}(t, y)$ is given by eq. (5.7). We can easily show that the above expression satisfies the fifth component of the equation for the conservation of energy (2.9) and that, in the limit $y \rightarrow \Delta$, the second term at the r.h.s. of eq. (5.8) vanishes, thus, ensuring the continuity of $\hat{T}_{5}^{5}$ across the boundary.

There is one remaining function we must determine, and that is the scale factor on the boundary of the brane, $a_{\Delta}(t)$. Again, we leave the derivation to Appendix, and simply display the result here

$$
a_{\Delta}(t)=a_{0}(t)\left[1-\frac{\hat{\kappa}^{2}}{12} \rho(t, 0) \Delta b\right] .
$$

The above result reveals the fact that the scale factor $a(t, y)$ decreases as we move away from the origin. Nevertheless, the rate of decrease being simply proportional to the energy 
density on the brane is much smaller than in the case of the Randall-Sundrum thin-wall solution [5] where the rate of decrease depends exponentially on the bulk cosmological constant. Due to the continuity condition on $a^{\prime}(t, y)$ at the boundary, the scale factor will also decrease mildly outside the brane until it reaches its unique minimum at $y= \pm\left|y_{\min }\right|$. The mild decrease of the scale factor makes necessary the compactification of the extra dimension which is realized through the identification of the two stable extrema $\pm\left|y_{\min }\right|$.

In this section, we have demonstrated that the positive features exhibited by the thinwall solution found in [19] also characterize its thick-wall analog. The cosmological solutions that emerge respect the 4-dimensional Friedmann equation on the brane and the compactification of the extra dimension takes place without the need for a second brane. In both instances, the existence of a non-vanishing $\hat{T}_{5}^{5}$ plays an important role as it results from the stabilization the extra dimension and helps restore standard cosmology on the brane.

\section{Conclusions}

While it may seem that standard FRW cosmology should arise trivially on a homogeneous 3-brane embedded in a higher dimensional space time, it has become clear that this result is highly dependent on the generation of a dilaton potential which fixes the dilaton expectation value and hence the size of the extra dimension. In the absence of a dilaton potential, there are two serious problems which must be overcome. Aside from the non-compact solution of Ref. [6], compactification of the extra-dimension in solutions for 3-branes with matter require the presence of a second brane on which the matter is constrained by Einstein's equations to take the form $\rho_{2}=-\rho_{1}+O\left(\rho^{2}\right)$. That is, we are forced to tolerate negative energy densities on the second brane. This is most assuredly unphysical. However, even if we accept this solution which includes the negative-brane, the solutions to Einstein's equations yield a cosmological expansion for which the Hubble parameter is proportional to the energy density. $\mathrm{BBN}$ and post BBN-cosmology can not be reconciled with this expansion law. We stress that we distinguish here the difference between a brane with negative tension and one with negative matter density. While a negative tension brane may naturally appear in string theory, we are not aware of any formulation which allows for a negative matter density $-\rho$.

In our previous work, we proposed that both of the aforementioned problems were tied to explicit assumptions made on the form of the energy-momentum tensor. Namely, that

$$
\hat{T}_{N}^{M}=\frac{\delta(y)}{b_{0}} \operatorname{diag}(-\rho, p, p, p, 0) .
$$

i.e., that the (55)-component of the energy-momentum tensor vanished. In [19], we showed that by allowing $\hat{T}_{55}$ to differ from zero, we could find a solution for the 3 -space scale factor 
$a(t, y)$, in the bulk, which has a local minimum at some co-ordinate value $y_{\text {min }}$, at which point we can compactify the extra dimension without the introduction of a second brane and in particular without a negative energy brane of any kind. Secondly, we derived the form of $\hat{T}_{5}^{5}$, in order to recover a normal Friedmann expansion on the 3-brane.

Here we have justified the choice for $\hat{T}_{5}^{5}$, and showed that it is the natural result of the back-reaction of a stabilized dilaton to the 3-brane with matter. In particular we have showed that the dilaton couples to the combination $\hat{T}_{\mu}^{\mu}-2 \hat{T}_{5}^{5}$, and any stable dilaton configuration requires this combination of energy-momentum components to vanish when averaged over the extra dimension. Furthermore, when a dilaton potential, which fixes the dilaton vev is included in the derivation of the solution, matter on the 3-brane will induce a shift in the dilaton vev in such a way so as to exactly guarantee the vanishing of $\hat{T}_{\mu}^{\mu}-2 \hat{T}_{5}^{5}$, and produce the needed (55)-component derived in [19].

We have, in addition, supplied generalization of our 3-brane solutions to include a cosmological constant both in the bulk and on the brane. We have considered the cases of negative, positive and zero bulk cosmological constant and demonstrated that a solution with a stable, compact extra dimension, which respects the standard 4D Friedmann equation, arises in each case. Moreover, the correct sign in the Friedmann equation is always maintained for every sign of $\Lambda_{B}$ as long as the sign of $\Lambda_{b r}$ is exactly opposite. In this case, the contributions of the two cosmological constants to the energy density of the universe can be cancelled leaving a term linear in $\rho$ to govern the expansion of the brane scale factor. Finally, our single-brane thin-wall solution derived in [19] was extended in the case of a brane-universe with a finite thickness. The resulting cosmological solution allows for the compactification of the extra dimension through the identification of the two stable minima of the scale factor outside the brane and the 4D Friedmann equation is once again recovered. In all of the above cases, the existence of a non-trivial fifth component of the energy-momentum tensor is necessary for the stabilization of the size of the extra dimension.

Acknowledgments We would like to thank G. Ross, V. Rubakov, M. Shifman and M. Voloshin for useful discussions. This work was supported in part by the Department of Energy under Grant No. DE-FG-02-94-ER-40823 at the University of Minnesota. The work of I.I.K. is supported in part by PPARC rolling grant PPA/G/O/1998/00567, the EC TMR grant FMRX-CT-96-0090 and by the INTAS grant RFBR - 950567.

\section{Appendix}

In this Appendix, we show the details of the derivation of the energy-momentum tensor inside the brane and subsequently generalize this solution for an arbitrary equation of state. 
For reasons, that will become obvious, we may rewrite the expression for the scale factor outside the brane (5.1) as follows

$$
a^{2}(t, y)=\frac{b^{2}}{\lambda^{2}(t)}\left[|y|+C_{\text {out }}(t)\right]^{2}-\frac{\lambda^{2}(t) E_{\text {out }}(t)}{2 b^{2}},
$$

where

$$
C_{\text {out }}(t)=\frac{\lambda^{2}}{2 b^{2}} C(t)=-\left|y_{\text {min }}\right|, \quad E_{\text {out }}(t)=2 b^{2}\left[\frac{b^{2}}{\lambda^{4}}\left(\Delta-\left|y_{\text {min }}\right|\right)^{2}-\frac{a_{\Delta}^{2}}{\lambda^{2}}\right] .
$$

To obtain the inside solution for the scale factor $a(t, y)$, we will assume, at first, that the pressure on the brane is zero, so our results would apply for a matter-dominated universe. Then, the zeroth-component of the equation for the conservation of energy (2.8) gives $\hat{\rho}=\hat{\rho}_{0} / a^{3}$. Integrating the (00)-component of Einstein's equations, the general solution for the scale factor takes the implicit form

$$
\begin{aligned}
\frac{2 A^{2}}{B^{3}(t)} \log \left(\frac{2}{B(t)}\right. & {\left.\left[B^{2}(t) a(t, y)-A^{2}\right]+2 \sqrt{E_{\text {in }}(t)+B^{2}(t) a^{2}(t, y)-2 A^{2} a(t, y)}\right) } \\
& +\frac{2}{B^{2}(t)} \sqrt{E_{i n}(t)+B^{2}(t) a^{2}(t, y)-2 A^{2} a(t, y)}= \pm \sqrt{2}\left[|y|+C_{i n}(t)\right]
\end{aligned}
$$

where

$$
A^{2}=\frac{2 b^{2} \hat{\kappa}^{2} \hat{\rho}_{0}}{3} . \quad B^{2}(t)=\frac{2 b^{2}}{\lambda^{2}(t)} .
$$

This is the same expression as the one that we derived in the thick-wall approximation [19] for the scale factor on the brane. The function $C_{i n}(t)$ can be determined by evaluating the solution at $y=0$, and has the form

$$
C_{i n}(t)= \pm \frac{\sqrt{2} A^{2}}{B^{3}(t)} \log \left(\frac{2}{B(t)}\left[B^{2}(t) a_{0}(t)-A^{2}\right]\right) .
$$

The remaining unknown function $E_{i n}(t)$ will be determined from the continuity of the first derivative of $a(t, y)$ with respect to $y$ at the boundary $y=\Delta$ between the brane and the bulk universe. If we use the form (A.1) for the solution outside the brane, this condition can be written as

$$
\pm \sqrt{2} a_{\Delta} a_{\Delta}^{\prime}=\sqrt{E_{\text {in }}(t)+B^{2}(t) a_{\Delta}^{2}-2 A^{2} a_{\Delta}}=\sqrt{E_{\text {out }}(t)+B^{2}(t) a_{\Delta}^{2}}
$$

leading to the result

$$
E_{i n}(t)=2 b^{2}\left[\frac{b^{2}}{\lambda^{4}}\left(\Delta-\left|y_{m i n}\right|\right)^{2}-\frac{a_{\Delta}^{2}}{\lambda^{2}}+\frac{2 \hat{\kappa}^{2}}{3} \hat{\rho}_{0} a_{\Delta}\right] .
$$


Moreover, the function $E_{i n}(t)$ is constrained by the (55)-component of Einstein's equations. When the solution (A.3) is substituted in eq. (2.6), we obtain the following constraint

$$
\frac{d E_{i n}(t)}{d t}=\frac{4 b^{2}}{3} \hat{\kappa}^{2} a^{3} \dot{a}\left(\hat{T}_{5}^{5}+\hat{\rho}\right) .
$$

This is satisfied if, and only if, the fifth component of the energy-momentum tensor, $\hat{T}_{5}^{5}$, on the brane is given by the expression in (5.8).

We have one more condition that our solution on the brane needs to satisfy, namely, the vanishing of $a^{\prime}(t, y)$ at the origin. This leads to the result

$$
E_{\text {in }}(t)=2 A^{2} a_{0}(t)-B^{2}(t) a_{0}^{2}(t) .
$$

If we substitute the expression for $E_{i n}(t)$ (A.7) in the above equation, we find the following constraint

$$
\frac{b^{2}}{\lambda^{2}}\left(\Delta-\left|y_{\min }\right|\right)^{2}=\frac{\left(a_{0}-a_{\Delta}\right)}{\Delta}\left[2 a_{0}\left|y_{\min }\right|-\Delta\left(a_{0}+a_{\Delta}\right)\right]
$$

where we have set $\hat{\rho}_{0}=\rho_{0} / 2 \Delta b$. In the limit $\Delta \rightarrow 0$, the above constraint should reduce to the expression (5.2) for $\left|y_{\text {min }}\right|$. This condition allows us to determine the function $a_{\Delta}(t)$ given in (5.9).

Finally, we extend the above analysis for the determination of the thick-wall solution inside the brane, for a matter-dominated universe, to the case of a brane-universe with a general equation of state $p=w \rho$. In this case, the equation for the conservation of energy gives the result $\hat{\rho}=\hat{\rho}_{0} / a^{3(1+w)}$. The first derivative of the scale factor on the brane, now, takes the form

$$
a^{\prime}(t, y)= \pm \frac{1}{\sqrt{2} a} \sqrt{E_{i n}(t)+B^{2}(t) a^{2}(t, y)-\frac{2}{(1-3 w)} A^{2} a(t, y)^{(1-3 w)}}
$$

and its continuity across the boundary $y=\Delta$ leads to the following result

$$
E_{\text {in }}(t)=2 b^{2}\left[\frac{b^{2}}{\lambda^{4}}\left(\Delta-\left|y_{\text {min }}\right|\right)^{2}-\frac{a_{\Delta}^{2}}{\lambda^{2}}+\frac{2 \hat{\kappa}^{2}}{3(1-3 w)} \hat{\rho}_{0} a_{\Delta}^{(1-3 w)}\right] .
$$

The constraint (A.8) that follows from the (55)-component of Einstein's equations still holds and leads to the following expression for the value of $\hat{T}_{5}^{5}$ on the brane

$$
\hat{T}_{5(b r)}^{5}(t, y)=\hat{T}_{5(B)}^{5}(t, y)+\hat{\rho}\left[\frac{n(t, \Delta)}{n(t, y)}\left(\frac{a(t, y)}{a(t, \Delta)}\right)^{3 w}-1\right]
$$

As in the previous case, we still have to fulfill the vanishing condition of $a^{\prime}(t, y)$ at the origin. Then, we find the result

$$
\frac{b^{2}}{\lambda^{2}}\left(\Delta-\left|y_{\min }\right|\right)^{2}=\frac{1}{\Delta}\left\{\frac{2\left|y_{\min }\right| a_{0}^{2}}{1-3 w}\left[1-\left(\frac{a_{0}}{a_{\Delta}}\right)^{3 w-1}\right]-\Delta\left(a_{0}^{2}-a_{\Delta}^{2}\right)\right\},
$$


which, in the limit $\Delta \rightarrow 0$, reduces to

$$
\left(\frac{a_{\Delta}}{a_{0}}\right)^{1-3 w}=1-\frac{\hat{\kappa}^{2}}{12} \rho(t, 0) \Delta b(1-3 w) .
$$

Note that for every value of $w$, larger or smaller that $1 / 3$, the scale factor decreases as we move away from the origin, thus, ensuring the monotonic behavior from the point $y=0$ to its minimum at $y=\left|y_{\min }\right|$.

However, the above expression gives a trivial result for the choice $w=1 / 3$ which corresponds to a radiation-dominated universe. Moreover, the expression (A.11) for the first derivative of $a(t, y)$ diverges for the same choice. This means that we have to study this case separately. Starting all over again and setting $\hat{\rho}=\hat{\rho}_{0} / a^{4}$, the first derivative, $a^{\prime}(t, y)$, takes the form

$$
a^{\prime}(t, y)= \pm \frac{1}{\sqrt{2} a} \sqrt{E_{\text {in }}(t)+B^{2}(t) a^{2}(t, y)-2 A^{2} \ln a(t, y)} .
$$

The result for $E_{i n}(t)$ is accordingly modified and is given by

$$
E_{i n}(t)=2 b^{2}\left[\frac{b^{2}}{\lambda^{4}}\left(\Delta-\left|y_{m i n}\right|\right)^{2}-\frac{a_{\Delta}^{2}}{\lambda^{2}}+\frac{2 \hat{\kappa}^{2}}{3} \hat{\rho}_{0} \ln a_{\Delta}\right],
$$

leading to the following form of $\hat{T}_{5}^{5}$ on the brane

$$
\hat{T}_{5(b r)}^{5}=\hat{T}_{5(B)}^{5}+\hat{\rho}\left[\frac{n(t, \Delta)}{n(t, y)} \frac{a(t, y)}{a(t, \Delta)}-1\right]
$$

Finally, the vanishing condition on $a^{\prime}(t, y)$ at the origin leads to the constraint

$$
\frac{b^{2}}{\lambda^{2}}\left(\Delta-\left|y_{\text {min }}\right|\right)^{2}=\frac{1}{\Delta}\left\{2\left|y_{\text {min }}\right| a_{0}^{2} \ln \left(\frac{a_{0}}{a_{\Delta}}\right)-\Delta\left(a_{0}^{2}-a_{\Delta}^{2}\right)\right\} .
$$

In the limit $\Delta \rightarrow 0$, we obtain the result

$$
a_{\Delta}(t)=a_{0}(t) \exp \left(-\frac{\hat{\kappa}^{2}}{12} \rho(t, 0) \Delta b\right) .
$$

Once again, the scale factor on the brane is decreasing, as $y$ increases, resulting in a similar behavior outside the brane until its minimum.

\section{References}

[1] E. Witten, Nucl. Phys. B471 (1996) 135;

P. Horava, and E. Witten, Nucl. Phys. B460 (1996) 506; B475 (1996) 94;

T. Banks and M. Dine, Nucl. Phys. B479 (1996) 173. 
[2] I. Antoniadis, Phys. Lett. B246 (1990) 377;

I. Antoniadis and K. Benakli, Phys. Lett. B326 (1994) 69;

I. Antoniadis, K. Benakli and M. Quiros, Phys. Lett. B331 (1994) 313;

I. Antoniadis, N. Arkani-Hamed, S. Dimopoulos and G. Dvali, Phys. Lett. B436 (1998) 257 ;

K. Dienes, E. Dudas and T. Gherghetta, Phys. Lett. B436 (1998) 55; Nucl. Phys. B537(1999) 47.

[3] N. Arkani-Hamed, S. Dimopoulos and G. Dvali, Phys. Lett. B429 (1998) 263.

[4] N. Arkani-Hamed, S. Dimopoulos and G. Dvali, Phys. Rev. D59 (1999) 086004.

[5] L. Randall and R. Sundrum, Phys. Rev. Lett. 83 (1999) 3370.

[6] L. Randall and R. Sundrum, hep-th/9906064.

[7] M.J. Duff, P. Hoxha, H. Lu, R.R. Martinez-Acosta, and C.N. Pope, Phys. Lett. B451 (1999) 38.

[8] A. Lukas, B.A. Ovrut, K.S. Stelle and D. Waldram, Phys.Rev. D59 (1999) 086001;

A. Lukas, B.A. Ovrut and D. Waldram, Phys. Rev. D60 (1999) 086001; hepth/9902071.

[9] N. Kaloper and A. Linde, Phys. Rev. D59 (1999) 101303.

[10] P. Binétruy, C. Deffayet and D. Langlois, hep-th/9905012.

[11] T. Shiromizu, K. Maeda and M. Sasaki, gr-qc/9910076.

[12] T. Nihei, Phys. Lett. B465 (1999) 81;

N. Kaloper, Phys. Rev. D60 (1999) 123506.

[13] C. Csáki, M. Graesser, C. Kolda and J. Terning, Phys. Lett. B462 (1999) 34.

[14] J.M. Cline, C. Grojean and G. Servant, Phys. Rev. Lett. 83 (1999) 4245.

[15] H.B. Kim and H.D. Kim, hep-th/9909053.

[16] E. Flanagan, S.H.H. Tye and I. Wasserman, hep-ph/9910498.

[17] D. Ida, gr-qc/9912002.

[18] C. Grojean, J. Cline and G. Servant, hep-th/9910081;

P. Kraus, hep-th/9910149;

A. Kehagias and E. Kiritsis, JHEP 9911 (1999) 022. 
[19] P. Kanti, I. Kogan, K.A. Olive and M. Pospelov, Phys. Lett. B468 (1999) 31.

[20] B.A. Campbell and K.A. Olive, Phys. Lett. B345 (1995) 429;

L. Bergstrom, S. Iguri, and H. Rubinstein, Phys. Rev. D60 (1999) 045005.

[21] U. Ellwanger, hep-th/9909103.

[22] W.D. Goldberger and M.B. Wise, Phys. Rev. D60 (1999) 107505; hep-ph/9907447; hep-ph/9911457.

[23] C. Csáki, M. Graesser, L. Randall, and J. Terning, hep-ph/9911406.

[24] P. Binétruy, C. Deffayet, U. Ellwanger and D. Langlois, hep-th/9910219. 